\title{
A Retrospective Comparison Study of Ultrasonography and Computed Tomography Scan in Diagnosis of Acute Appendicitis in the Pediatric Population
}

\author{
Vincent Marcucci ${ }^{\mathrm{a}}$, Stephanie Yee ${ }^{\mathrm{b}, \mathrm{e}}$, Roberto C. Castillo ${ }^{\mathrm{c}}$, \\ Elias N. Fakhoury ${ }^{\mathrm{d}}$, Jamshed Zuberi ${ }^{\mathrm{b}}$
}

\begin{abstract}
Background: Ultrasonography (US) has largely become the primary diagnostic imaging modality for the diagnosis of acute appendicitis (AA) in pediatric patients. The purpose of this study was to determine and compare the diagnostic accuracy of helical computed tomography $(\mathrm{CT})$ scans and graded compression US for the diagnosis of AA in the pediatric population.
\end{abstract}

Methods: Between January 2011 and December 2013, 431 pediatric patients (aged 5 - 18 years) who presented with acute abdominal pain and received either a CT scan, US, or both for the diagnosis of AA were retrospectively reviewed from an IRB-approved institutionwide database. Sensitivities and specificities of both imaging modalities were calculated and compared.

Results: Patients were allocated into two cohorts depending on whether they received an US (239/431) or CT (192/431). Clinical symptoms and laboratory values were noted and analyzed for the significance of mesenteric lymphadenitis in conjunction with appendicitis and for differential diagnosis. A total of $182 \mathrm{CT}$ cases and 227 US cases were verified as appendicitis via histopathology report. The overall sensitivity and specificity of CT imaging were determined to be $91.2 \%$ and $70 \%$, respectively, while the sensitivity and specificity for US imaging were $52.8 \%$ and $83.3 \%$.

Conclusion: Helical CT imaging in the pediatric population has provided a higher sensitivity and diagnostic accuracy for AA. However, the diagnostic benefit of US cannot be excluded in this patient population. Further research is needed on whether CT imaging should be used primarily for the diagnostic approach to AA in pediatric patients

Manuscript submitted March 19, 2020, accepted July 21, 2020

Published online August 22, 2020

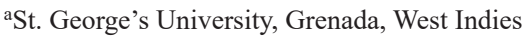

bSt. Joseph's University Medical Center, Paterson, NJ, USA

'St. Luke's University Hospital, Bethlehem, PA, USA

${ }^{d}$ Buffalo General Medical Center, Buffalo, NY, USA

${ }^{e}$ Corresponding Author: Stephanie Yee, Department of Surgery, St. Joseph's

University Medical Center, 703 Main Street, Paterson, NJ 07504, USA.

Email: syee.123@gmail.com

doi: https://doi.org/10.14740/jcs404 complaining of acute abdominal pain.

Keywords: Acute appendicitis; Ultrasonography; Computed tomography; Acute abdominal pain

\section{Introduction}

Acute abdominal pain is one of the most common complaints in the pediatric population presenting to the emergency department. It is usually a symptom of a self-limiting condition such as constipation, mesenteric lymphadenitis, gastroenteritis, or a viral illness [1]. There is an ongoing challenge for physicians to distinguish severe, potentially life-threatening conditions such as appendicitis, and whether further evaluation and emergent treatment is necessary $[1,2]$. Accurate diagnosis of acute appendicitis (AA) in pediatric patients further complicates the challenge for physicians due to the presentation of atypical symptoms [3]. At our institution, ultrasonography (US), used in conjunction with a thorough history and physical exam, is often the primary diagnostic tool. While US is a relatively safe option for diagnosing AA in children due to the lack of radiation exposure, literature has shown equivocal findings and the need for a better and more definitive diagnostic approach [4].

Ongoing research to utilize more accurate and reliable methods to correctly diagnose appendicitis has been challenging. The use of computed tomography (CT) for appendicitis has shown promise with slightly higher sensitivity and specificity as a diagnostic approach. In a meta-analysis conducted in 2007 comparing the diagnostic accuracies of CT vs. US in adult patients with appendicitis, CT scans provided a higher sensitivity ( $88.4 \%$ vs. $76 \%$ ) and specificity ( $90.4 \%$ vs. $89.4 \%)$ when compared to US $[4,5]$. However, there is concern whether this approach is suitable for children and adolescent patients due to the relatively high amount of radiation a child is exposed to when undergoing CT imaging [6].

A confounding illness associated with AA is mesenteric lymphadenitis (ML). ML can pose difficulty for emergency room (ER) physicians and surgeons when trying to diagnose AA. ML is an inflammatory response of the abdominal lymph nodes, commonly the para-aortic and ileo-cecal nodes causing pain localized to the right lower quadrant [7]. ML may be a 
sequela of a viral (rotavirus or norovirus) or bacterial (Yersinia enterocolitica) gastroenteritis, and a common occurrence in patients suffering from AA $[7,8]$.

The purpose of this study was to determine and compare the diagnostic accuracy of helical CT versus graded compression US for the diagnosis of appendicitis in a pediatric population (aged 5 - 18 years). The associated symptomatic complaints were considered and studied for the purpose of determining whether ML when present with AA versus AA alone will allow for a better, safer and more accurate diagnostic approach in the emergency department.

\section{Materials and Methods}

\section{Patient selection}

Between January 2011 and December 2013, 431 pediatric patients (5 - 18 years old) who presented to St. Joseph's Regional Medical Center's emergency department with acute abdominal pain who received either a CT scan or US for the diagnosis of appendicitis and ML were reviewed. This study was conducted as retrospective observational review approved by the St. Joseph's University Medical Center Institutional Review Board. Exclusion criteria included patients who were diagnosed with appendicitis, but did not present with acute abdominal pain, individuals not in the specified age range and patients who were determined to have developmental delay. Of the subjects included, the data collection per each patient comprised of their age, sex, additional symptoms (nausea, vomiting, diarrhea, constipation, upper respiratory infection: cough or congestion), laboratory findings (white blood cell (WBC) with differential and SMA-7), ER management (medication received and fluids received), radiological findings (US and CT) and histopathological reports.

\section{US and CT}

CT cases utilized a multi-view, $2.5-5.0 \mathrm{~mm}$ slice thickness (dependent on the size and weight in kilograms of the patient), with a 16-slice or 64-slice scanner. Contrast was given prior to imaging when the need for $\mathrm{CT}$ diagnosis was not determined to be emergent. Oral, IV, or rectal route of contrast administration was administered on a case dependent basis on whether the child could tolerate consuming oral contrast. General guidelines for diagnosing appendicitis via $\mathrm{CT}$ included a diameter greater than $6.5 \mathrm{~mm}$, with associated peri-appendiceal inflammation, fluid, or appendicoliths [9].

US cases involved a linear transducer to identify and access the quality and extent of disease to the appendix for the majority of patients. A curvilinear transducer was used in patients with a large body habitus in order to gain adequate depth. AA was diagnosed following the general guidelines of an outer appendicular diameter greater than $6-7 \mathrm{~mm}$, non-compressible (in non-perforated cases) and lack of peristalsis [10].

US and CTs were interpreted as positive, negative, or nondiagnostic for AA. Radiographic findings were correlated with
Table 1. Demographics and Radiologic Distributions

\begin{tabular}{ll}
\hline Patients $(\mathrm{n})$ & 431 \\
Age at operation, years (mean $\pm \mathrm{SD})$ & $12.29 \pm 3.87$ \\
Gender (male/female) & $259 / 172$ \\
CT/US cohorts & $192 / 239$ \\
$\quad$ CT (+) & 169 \\
CT (-/equivocal) & 23 \\
$\quad$ US (+) & 122 \\
$\quad$ US (-/equivocal) & 117 \\
\hline
\end{tabular}

CT/US (+) is indicative of positive appendicitis per radiology report. CT/ US (-/equivocal) is indicative of negative appendicitis, appendix not visible, or unclear on radiology report. SD: standard deviation; CT: computed tomography; US: ultrasound.

the histopathological reports after operative intervention. The sensitivity and specificity of each imaging modality was calculated and compared. Radiological diagnosis of ML was defined as three or more lymph nodes visible with a short axis diameter of $5 \mathrm{~mm}$ or greater located in the right lower quadrant [11].

\section{Statistical analysis}

SPSS version 18 and Graphpad version 6.0 were utilized for analyzing data. Numerical values of analyzed data were compared and displayed graphically. Sensitivities and specificities for CT and US were calculated using two-by-two tables. A P value of $<0.05$ was considered statistically significant.

\section{Results}

A total of 431 patients who underwent CT or US for the diagnosis of appendicitis were reviewed. Patients were designated into a CT cohort $(\mathrm{n}=192,44.5 \%)$ or a US cohort $(\mathrm{n}=239$, $55.5 \%$ ) (Table 1). All patients from both cohorts had a surgical specimen sent for an official histopathological report. A total of $182(94.8 \%)$ and $227(94.9 \%)$ of the cases within the CT and US cohorts respectively were verified as appendicitis through histopathological confirmation (Table 2). The sensitivity and specificity for CT was calculated, providing $91.2 \%$ and $63.3 \%$ respectively in regards to correctly diagnosed appendicitis when compared to pathology reports. The US cohort had an overall sensitivity and specificity calculated at $52.8 \%$ and $83.3 \%$, respectively (Table 2 ). The positive predictive values (PPVs) for both cohorts were relatively similar. The US cohort's PPV was calculated at $98.4 \%$, while the CT cohort's PPV was slightly lower at $97.6 \%$ (Table 2).

Of the 431 patients, 423 were evaluated for the incidence of $\mathrm{ML}$ in association with AA. In this patient population, 109 $(25.8 \%)$ were identified as positive for ML, while $314(74.2 \%)$ were diagnosed with AA without the incidence of ML (Table 2). Male gender was found to have a significantly higher incidence of AA $(\mathrm{n}=259,60.1 \%)$ when compared with females (n $=172,39.9 \%)$. Nearly half $(45.6 \%)$ of the patient population 
Table 2. Radiologic Accuracy in Conjunction With Pathology Report and Incidence of ML

\begin{tabular}{ll}
\hline CT/US cohorts & \\
CT sensitivity/specificity (\%)* & $91.16 \% / 63.63 \%$ \\
\hline CT PPV (\%) & $97.63 \%$ \\
\hline US sensitivity/specificity (\%)* & $52.86 \% / 83.33 \%$ \\
US PPV (\%) & $98.36 \%$ \\
Positive pathology (n, \%) & $182(94.8 \%)$ \\
CT & $227(94.9 \%)$ \\
US & $109(25.8 \%)$ \\
\hline ML (n, \%)
\end{tabular}

*Low number of subjects calculated for specificity which may not be congruent with available literature [5, 9]. PPV: positive predictive value; ML: mesenteric lymphadenitis; CT: computed tomography; US: ultrasonography.

who presented with acute abdominal pain between 13 and 18 years of age was diagnosed with AA. ML decreased significantly after the first decade of life. ML was confirmed in 35\% of the cases between ages 5 and 8 years, with the incidence reducing to $19 \%$ in patients between the ages of 13 and 18 years who presented with acute abdominal pain (Fig. 1).

Several clinical symptoms were analyzed in association for the occurrence of AA. The prevalence of vomiting without associated diarrhea or constipation was a statistically significant finding in this study population $(\mathrm{P}<0.05)$ (Fig. 2 ). There were equivocal laboratory values in patients with
AA; however, it was determined an elevation in segmented neutrophils was much more likely than leukocytosis (Fig. 3). Associated fever, whether measured at home or in the emergency department, was not statistically significant when diagnosing AA in pediatric patients presenting with acute abdominal pain (Fig. 4).

\section{Discussion}

The diagnostic approach to acute abdominal pain in pediatric patients has grown increasingly depending on imaging studies over the last two decades [12]. Technological advancement also places a financial impact on the health care services that are attainable and available for improving the quality of treatment $[12,13]$. While US remains a first-line method for diagnosing AA, in this study, 91 patients who had an appendix that was not visible, negative, or an unclear imaging report had a follow-up CT scan, where 85 of these patients were diagnosed with AA which was further validated with the pathologic findings. Figure 5 displays a normal appearing appendix $(<6 \mathrm{~mm}$ in diameter) on US in a 9-year-old child. Figures 6 and 7 show CT images of the same child showing a ruptured appendix with significant right lower quadrant fluid accumulation. The Alvarado scoring system consists of clinical and laboratory values used in an attempt to stratify patients into low, intermediate and high risk for appendicitis [14]. This scoring system was not utilized in our study due to several of the subjective criteria being unavailable upon retrospective analysis. However, the Alvarado scoring system may be useful in future studies for

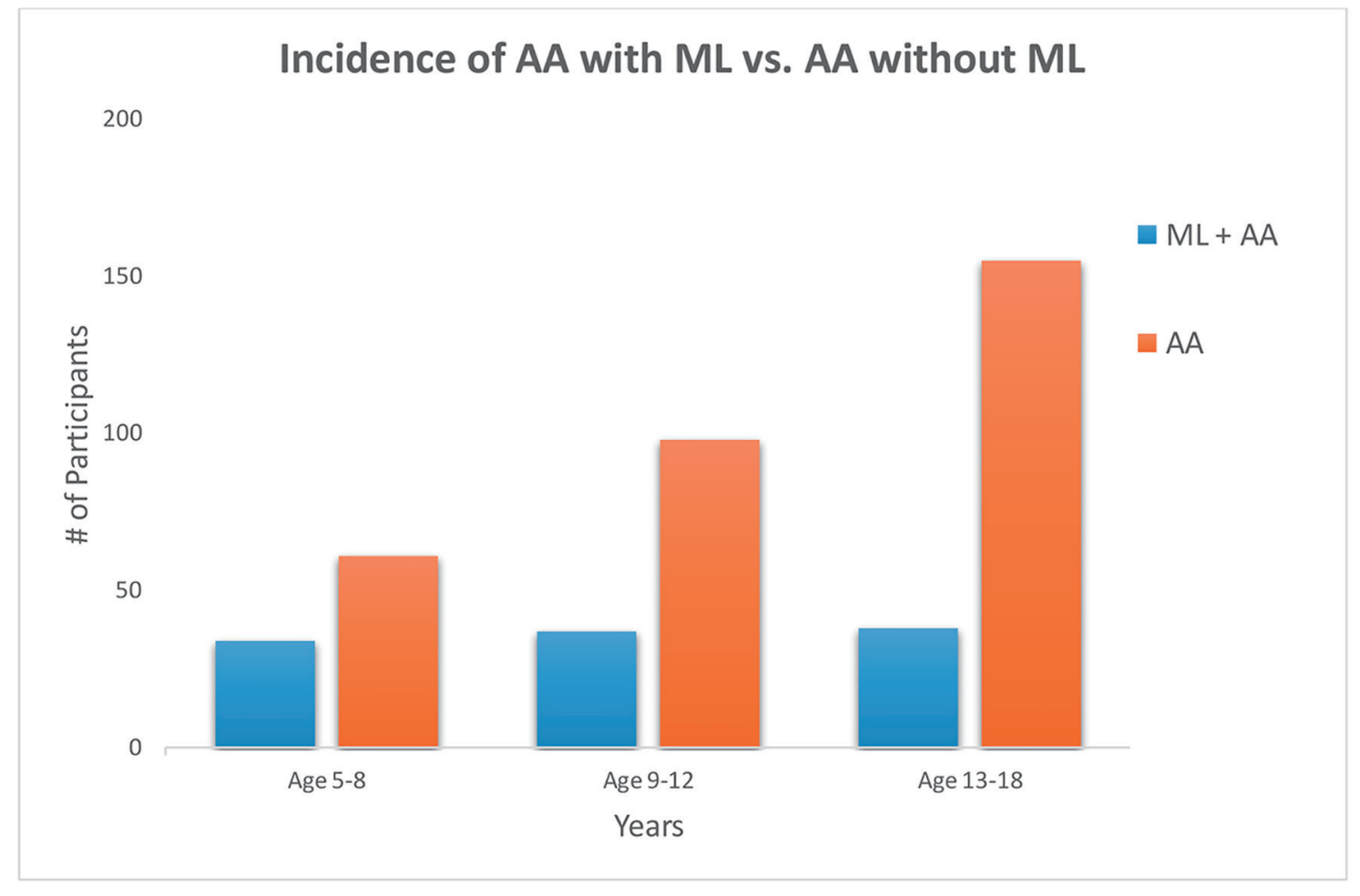

Figure 1. Incidence of $M L$ decreases significantly after the first decade of life $(P=0.012)$. ML: mesenteric lymphadenitis. 


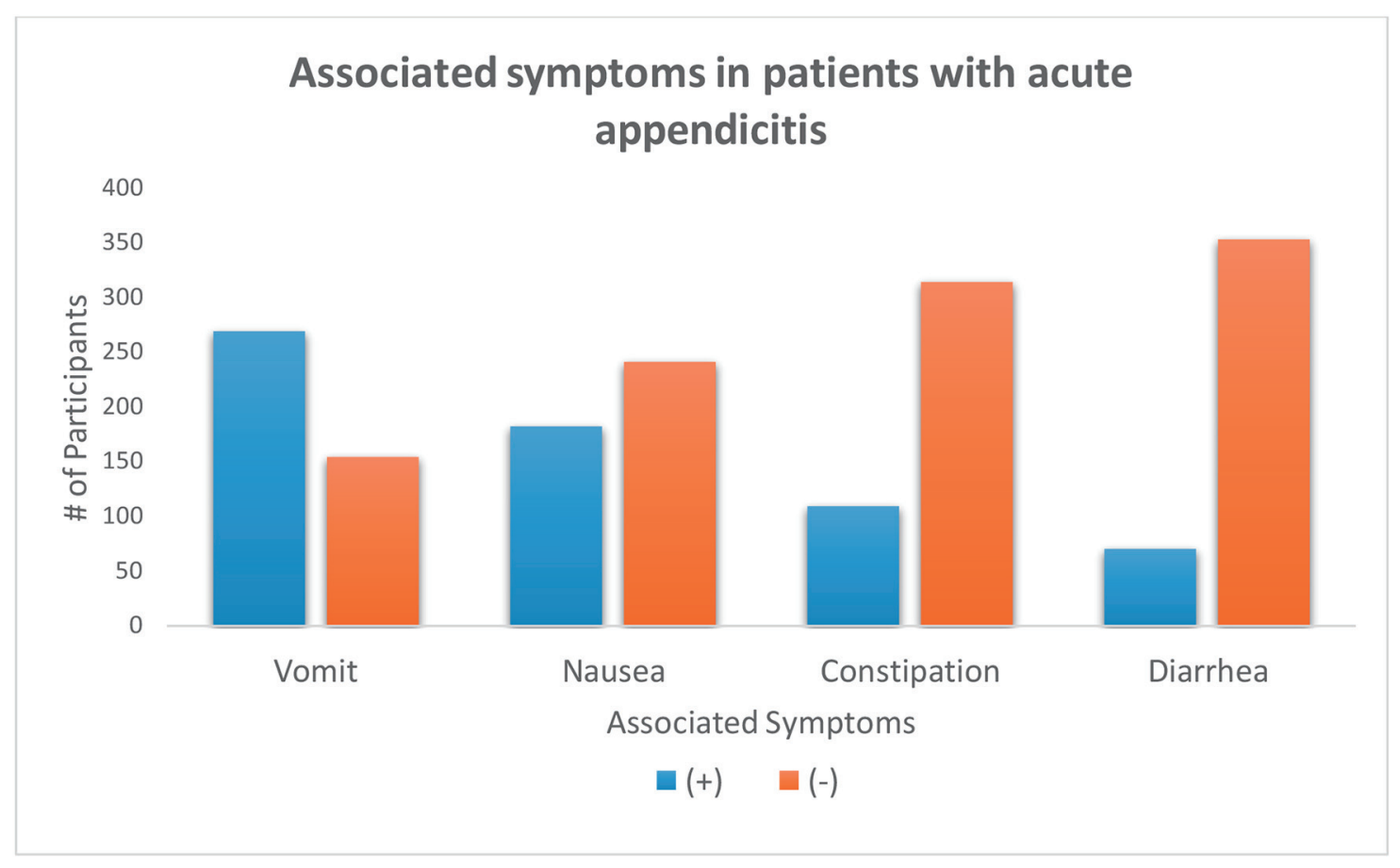

Figure 2. Vomiting is a likely symptom of acute appendicitis without the presentation of constipation or diarrhea $(P=0.0001)$. Subjective assessment for nausea as a symptom of acute appendicitis was insignificant $(P=0.056)$.

patients under the age of 13 where appendicitis is less likely and misdiagnosis is typically higher [15]. In emergent cases where a high Alvarado score is reported, physicians may be able to use this score as a method to triage patients [14]. In

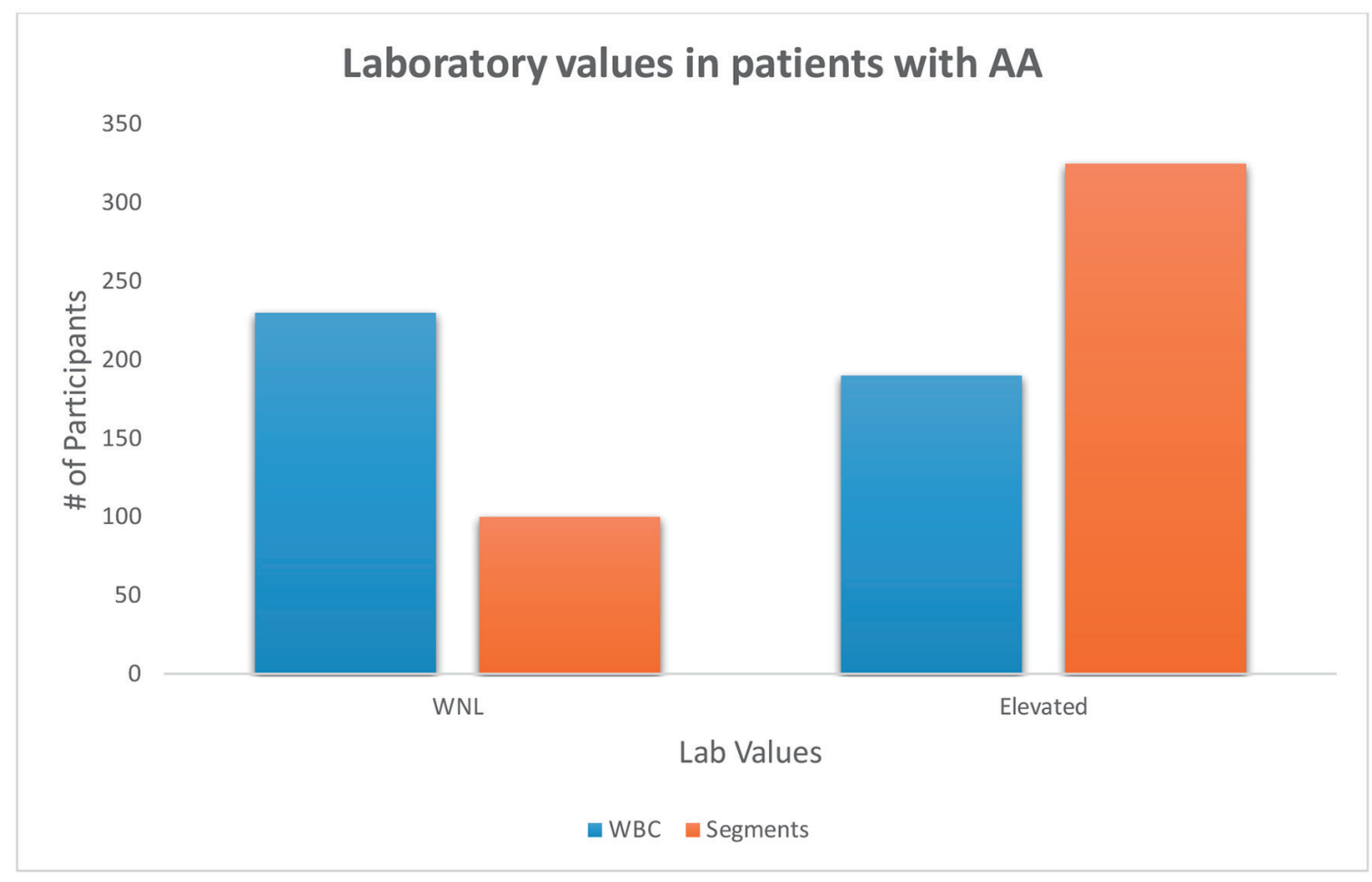

Figure 3. Patients diagnosed with acute appendicitis were more likely to have elevated segmented neutrophils than leukocytosis on complete blood cell $(P=0.006)$. WNL: within normal limit. 


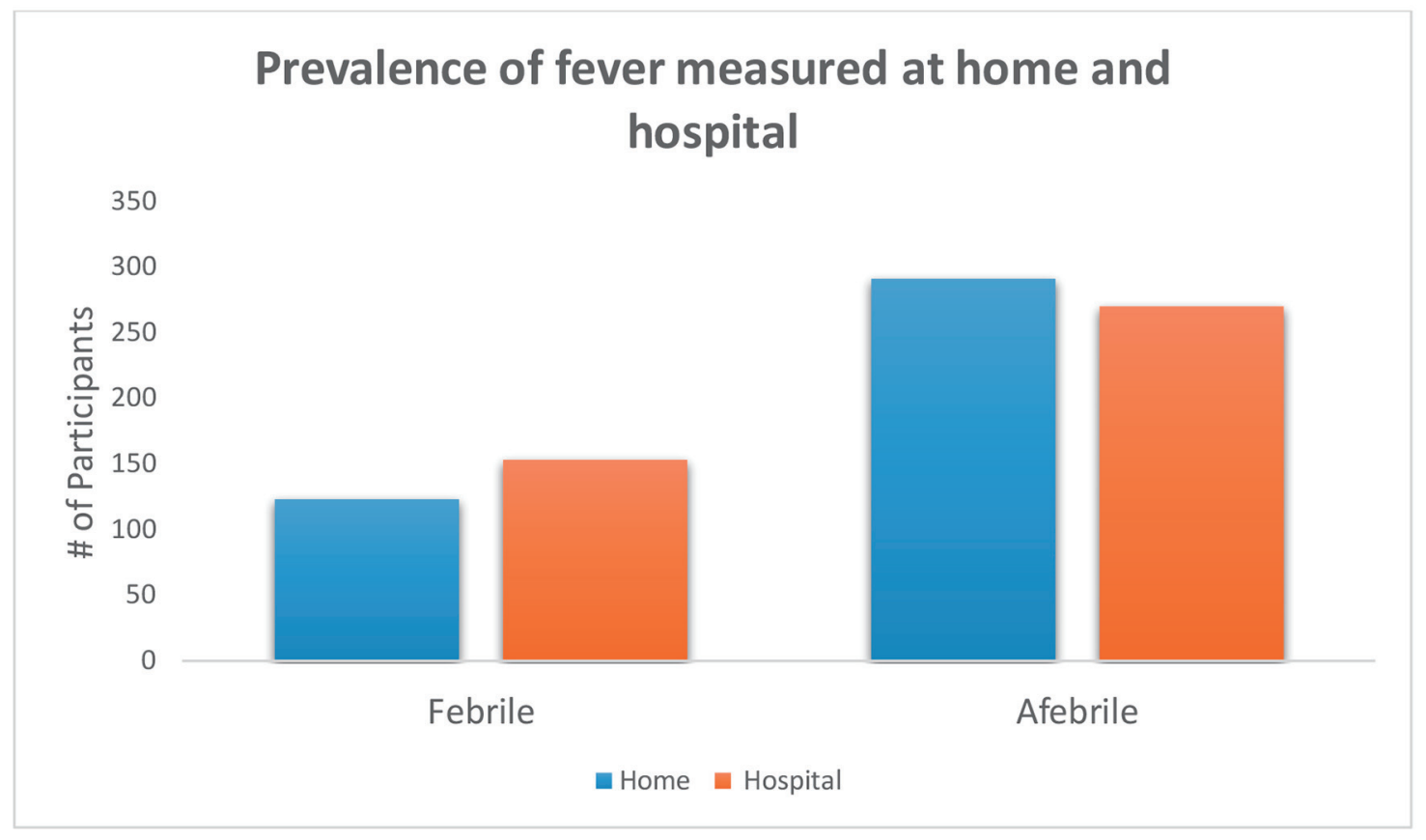

Figure 4. Insignificant findings in study population ( $P=0.615$ and 0.165 at home and in the hospital, respectively).

these cases, the use of a CT scan may supersede the initial imaging with US for improved diagnostic accuracy and preventing delays in surgical intervention.

Literature has supported higher sensitivity and specificity for CT imaging over US for diagnosing AA in adult patients [5, $9,10,12]$. There is minimal literature to support such claims in pediatric patients. The lower specificity for CT when compared with US in this study may have been influenced by a small patient population and further research is needed to support these findings. Further drawbacks to CT imaging such as cost effectiveness, radiation exposure and overall time constraints may prevent CT from being regarded as the first-line imaging modality in children with acute abdominal pain. The specificity and sensitivity of US and CT imaging remained consistent throughout the age range of 5 - 18 in this study. Subjective decision making on what imaging modality to utilize in the pediatric population for acute abdominal pain varies from case to case and may also be influenced on whether a general surgeon or ER physician was the ordering physician [12].

Differential diagnosis for acute abdominal pain is a crucial

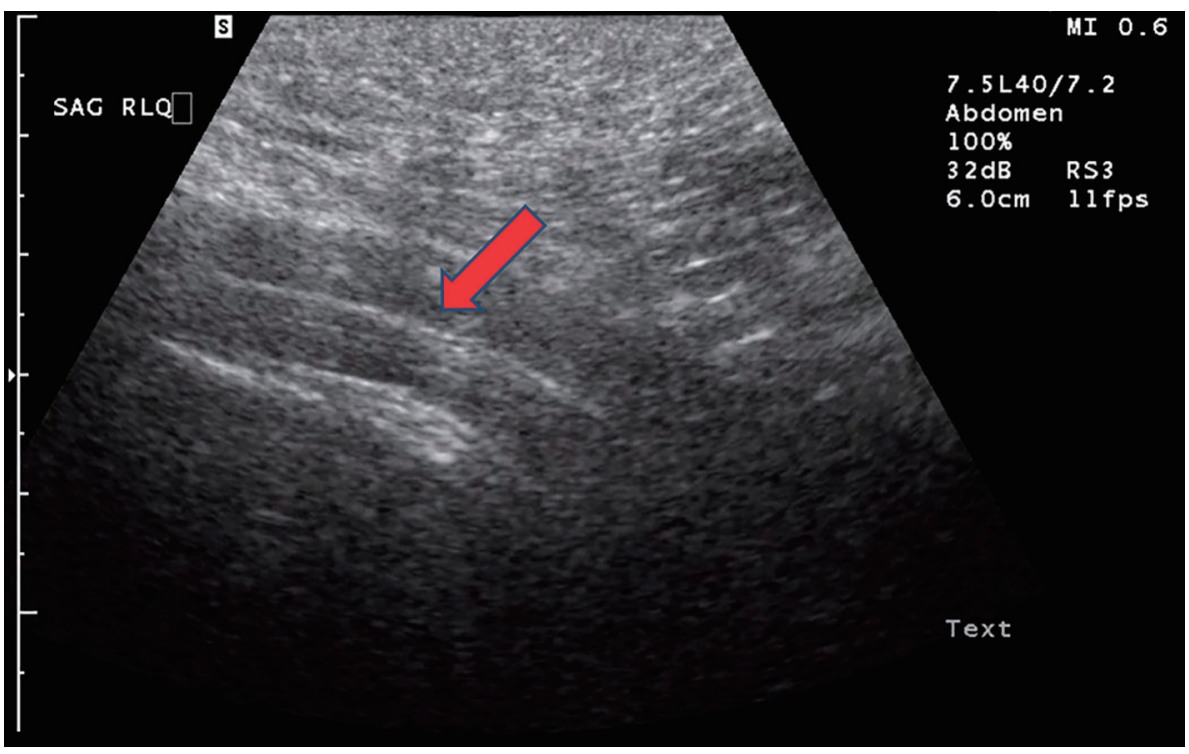

Figure 5. Abdominal US displaying a normal diameter $(<6 \mathrm{~mm})$ in a 9-year-old child with suspected appendicitis. Red arrow indicates appendix. US: ultrasonography 


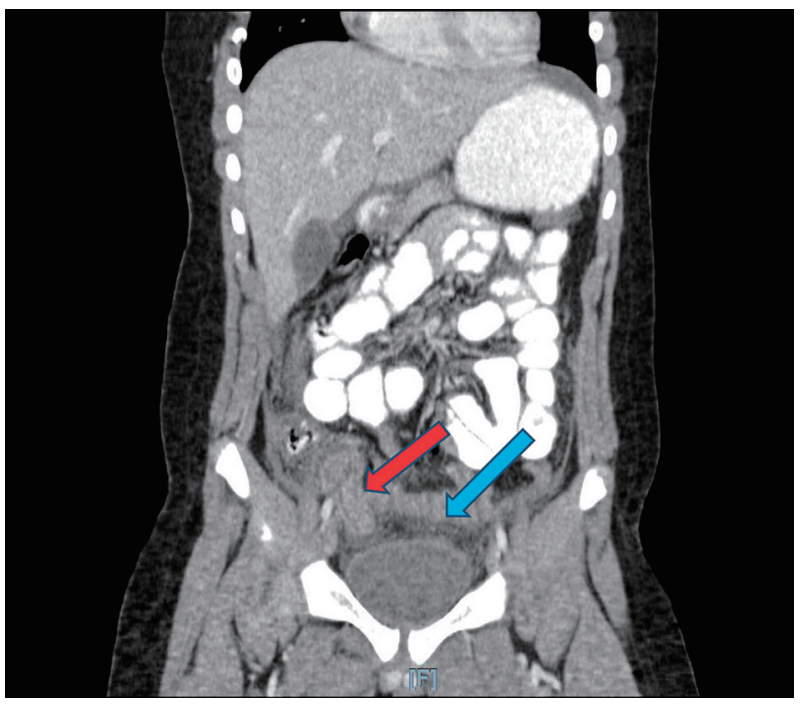

Figure 6. Coronal CT imaging of the same child suggesting ruptured appendicitis (red arrow) with extensive peritonitis in the pelvis and free fluid in the pelvis (blue arrow). CT: computed tomography.

aspect of determining the correct treatment for a patient and minimizing hospital length of stay and unwarranted future ER visits. The aim of this study was to evaluate the potential confounding ability of ML in patients complaining of abdominal pain and how likely it is to be present when a patient has AA. ML primarily occurs in children in their first decade of life and decreases significantly after the age of 20 [7, 8]. Our study was largely inconclusive in determining alarm symptoms for patients with $\mathrm{ML}$ when compared to AA and patients who had ML as a symptom of AA. This may be a consequence of subjectivity in patients' responses while taking a thorough history and whether or not ML was noted in the US and CT report.

Pediatric patients with AA have historically been misdiagnosed in $7.5-37 \%$ of the time [15]. Gastrointestinal symptoms such as nausea, vomiting, constipation and diarrhea are common complaints in pediatric ERs, and may be associated with acute gastroenteritis, the most common inflammatory condition within the abdomen in children $[15,16]$. In this study vomiting was found to be present in over $60 \%$ of patients; however, the finding of nausea was only reported in slightly over $40 \%$ of the cases. This discrepancy between nausea and vomiting may be attributed to nauseating symptoms being masked by abdominal pain. A previous 2007 study by Chang et al highlighted misdiagnosed cases of AA, in which $70 \%$ of those patients also had elevated WBC counts [15]. The advent of leukocytosis may be a helpful clue in accessing patients suspected of AA. Contradictory findings in our study showed only $45 \%$ of patients had elevated WBC counts above the normal range. The retrospective approach to this study limited the ability to analyze the length of clinical symptom complaint and abnormal laboratory values. Diagnostic accuracy for AA has been found to be $\geq 80 \%$ when leukocytosis is noted for longer than $49 \mathrm{~h} \mathrm{[15].}$

Limitations of this study include the retrospective research protocol, failure to consider cost effectiveness, as well as strat-

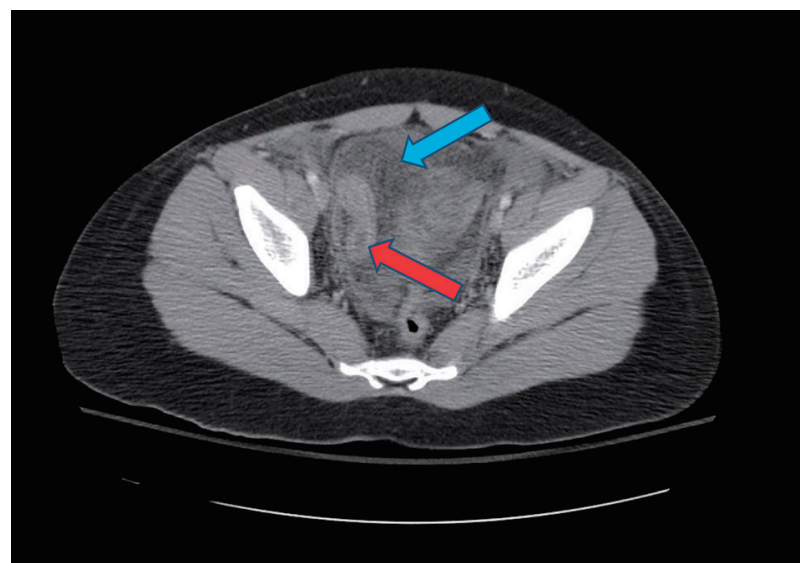

Figure 7. Transverse abdominal section of CT imaging consistent with ruptured appendicitis (red arrow) and significant pelvic fluid accumulation (blue arrow) previously missed on abdominal US. CT: computed tomography; US: ultrasonography.

ification of clinical symptoms and lab values, also, failure to consider the type of physician ordering the imaging tests, and whether a child received oral, IV, rectal, or non-contrast CT images. Lastly, potential adverse effects of radiation exposure may pose a limitation.

In conclusion, helical $\mathrm{CT}$ has been shown to have a significantly higher sensitivity and accuracy in diagnosing appendicitis in the pediatric population at our institution. However, this does not diminish the benefits of US in the diagnostic algorithm. The higher specificity seen in this study population demonstrates US is a useful tool in ruling out appendicitis in the appropriate clinical setting. US provides a short acquisition time and lack of ionizing radiation, which make it an ideal imaging modality for pediatric patients with suspected AA. However, further studies are needed to determine which cases of abdominal pain may warrant a CT scan instead of an US for the preliminary diagnosis of AA in pediatric patients.

\section{Acknowledgments}

St. Joseph’s University Medical Center.

\section{Financial Disclosure}

None to declare.

\section{Conflict of Interest}

None to declare.

\section{Informed Consent}

Not applicable. 


\section{Author Contributions}

Study concept and design: Castillo, Fakhoury, Yee, Zuberi. Acquisition of data: Castillo, Fakhoury. Analysis and interpretation of data: Castillo, Fakhoury, Marcucci, Yee, Zuberi. Statistical analysis: Castillo, Fakhoury, Marcucci, Yee, Zuberi. Drafting of manuscript: Marcucci, Yee. Critical revision: Castillo, Fakhoury, Zuberi.

\section{Data Availability}

The data supporting the findings of this study are available from the corresponding author upon reasonable request.

\section{References}

1. Kim JS. Acute abdominal pain in children. Pediatr Gastroenterol Hepatol Nutr. 2013;16(4):219-224.

2. Grant HW, Parker MC, Wilson MS, Menzies D, Sunderland G, Thompson JN, Clark DN, et al. Adhesions after abdominal surgery in children. J Pediatr Surg. 2008;43(1):152-156; discussion 156-157.

3. Scholer SJ, Pituch K, Orr DP, Dittus RS. Clinical outcomes of children with acute abdominal pain. Pediatrics. 1996;98(4 Pt 1):680-685.

4. Mostbeck G, Adam EJ, Nielsen MB, Claudon M, Clevert D, Nicolau C, Nyhsen C, et al. How to diagnose acute appendicitis: ultrasound first. Insights Imaging. 2016;7(2):255-263.

5. Al-Khayal KA, Al-Omran MA. Computed tomography and ultrasonography in the diagnosis of equivocal acute appendicitis. A meta-analysis. Saudi Med J. 2007;28(2):173-180.

6. Smith-Bindman R, Lipson J, Marcus R, Kim KP, Mahesh M, Gould R, Berrington de Gonzalez A, et al. Radiation dose associated with common computed tomography examinations and the associated lifetime attributable risk of cancer. Arch Intern Med. 2009;169(22):2078-2086.

7. Helbling R, Conficconi E, Wyttenbach $\mathrm{M}$, Benetti C, Simonetti GD, Bianchetti MG, Hamitaga F, et al. Acute nonspecific mesenteric lymphadenitis: more than "No Need for Surgery". Biomed Res Int. 2017;2017:9784565.

8. Vayner N, Coret A, Polliack G, Weiss B, Hertz M. Mesenteric lymphadenopathy in children examined by US for chronic and/or recurrent abdominal pain. Pediatr Radiol. 2003;33(12):864-867.

9. Vaghela K, Shah B. Diagnosis of acute appendicitis using clinical Alvarado scoring system and computed tomography (CT) criteria in patients attending Gujarat Adani Institute of Medical Science - a retrospective study. Pol J Radiol. 2017;82:726-730.

10. Benabbas R, Hanna M, Shah J, Sinert R. Diagnostic accuracy of history, physical examination, laboratory tests, and point-of-care ultrasound for pediatric acute appendicitis in the emergency department: a systematic review and meta-analysis. Acad Emerg Med. 2017;24(5):523551.

11. Rao PM, Rhea JT, Novelline RA. CT diagnosis of mesenteric adenitis. Radiology. 1997;202(1):145-149.

12. Yazici P, Oz A, Kartal K, Battal M, Kabul Gurbulak E, Akgun IE, Yetkin SG, et al. Emergency computed tomography for the diagnosis of acute appendicitis: How effectively we use it? Ulus Travma Acil Cerrahi Derg. 2018;24(4):311-315.

13. Kumar RK. Technology and healthcare costs. Ann Pediatr Cardiol. 2011;4(1):84-86.

14. Ohle R, O'Reilly F, O'Brien KK, Fahey T, Dimitrov BD. The Alvarado score for predicting acute appendicitis: a systematic review. BMC Med. 2011;9:139.

15. Chang YJ, Chao HC, Kong MS, Hsia SH, Yan DC. Misdiagnosed acute appendicitis in children in the emergency department. Chang Gung Med J. 2010;33(5):551-557.

16. Beltran MA, Almonacid J, Vicencio A, Gutierrez J, Cruces KS, Cumsille MA. Predictive value of white blood cell count and C-reactive protein in children with appendicitis. J Pediatr Surg. 2007;42(7):1208-1214. 